\title{
Formation of $s p^{3}$ Bonding in Nanoindented Carbon Nanotubes and Graphite
}

\author{
Wanlin Guo, ${ }^{1, *}$ C. Z. Zhu, ${ }^{1,2}$ T. X. Yu, ${ }^{2}$ C. H. Woo, ${ }^{3}$ B. Zhang, ${ }^{1}$ and Y.T. Dai ${ }^{1}$ \\ ${ }^{1}$ Institute of Nano Science, Nanjing University of Aeronautics and Astronautics, Nanjing 210016, China \\ ${ }^{2}$ Department of Mechanical Engineering, Hong Kong University of Science and Technology, Hong Kong, China \\ ${ }^{3}$ Department of Electronic and Information Engineering, Hong Kong Polytechnic University, Hong Kong, China
}

(Received 19 April 2004; published 8 December 2004)

\begin{abstract}
Nanoindentation-induced interlayer bond switching and phase transformation in carbon nanotubes (CNTs) and graphite are simulated by molecular dynamics. Both graphite and CNTs experience a softto-hard phase transformation at room temperature at compressive stresses of 12 and $16 \mathrm{GPa}$, respectively. Further penetration leads to the formation of interlayer $s p^{3}$ bonds, which are reversible upon unloading if the compressive stress is under about $70 \mathrm{GPa}$, beyond which permanent interlayer $s p^{3}$ bonds form. During nanoindentation, the maximum nanohardness of graphite can reach $109 \mathrm{GPa}$, and CNTs $120 \mathrm{GPa}$, which is comparable to that of diamond.
\end{abstract}

DOI: $10.1103 /$ PhysRevLett.93.245502

Carbon exists in many distinct forms, such as graphite, diamond, fullerenes, and carbon nanotubes (CNTs). In graphite, the carbon atoms are arranged in a layered structure of hexagonal rings with hybridized $s p^{2}$ atomic bonding. Carbon atoms can also form $s p^{3}$ bonds with the four nearest neighbors, creating the pyramidal lattice of a superhard cubic-diamond crystal. Similar to graphite, the fullerenes and CNTs have the hexagonal ring structure bound by $s p^{2}$ bonds. However, these materials, particularly the CNTs, take on a variety of lattice structures that exhibit different mechanical and electric properties [1,2]. In the last decade, many high-pressure experiments have revealed the fascinating phase transformation of fullerenes into superhard polymeric and disordered-amorphous carbon phases. The hardness sometimes can approach that of single-crystal diamond [3-8], even at room temperature $[9,10]$. On the other hand, in graphite subjected to high pressure up to $65 \mathrm{GPa}$ at room temperature, no superhardness is found after the pressure is removed [10,11]. Recently, a new phase hard enough to crack diamond anvils is obtained from graphite undergoing a transition of $s p^{2}$ to $s p^{3}$ bonding at a pressure of about $17 \mathrm{GPa}$ [12]. The graphitelike hexagonal boron nitride can also form $s p^{3}$ bonding under compressive loading [13].

Perfect chiral CNTs are radically different from graphite sheets and diamond crystals, in that unpaired electrons in a CNT cause one-dimensional superconductivity or semiconductivity, depending on the chiral structure [14]. As a result, its conductivity can be tuned by mechanical deformations [15], sometimes even into single electron transistors [16]. The unpaired electrons in CNTs also facilitate bond formation with other molecules or atoms, and produce tunable electric properties, which cause the CNT to function as nanosensors [17]. Crosslinks by $s p^{3}$ bondings can increase the bending modulus of CNT bundles by 30-fold [18]. Therefore, phase transition and/or bond switching in CNTs under compression may have significant potential in nanotechnology. Geometrical deformation of CNT bundles under pressure
PACS numbers: 62.25.+g, 62.50.+p, 81.05.Uw, 81.07.De

of up to several GPa have been widely studied $[19,20]$, and the lattice structure of the tube lattice may collapse when the pressure is over about $4 \mathrm{GPa}$ [21]. When singlewalled CNT samples are subjected to high pressures of between 3 and $42 \mathrm{GPa}$, the electrical resistance shows a steady increase, followed by a sharp rise when the pressure exceeds $42 \mathrm{GPa}$ [22]. However, in first-principles calculations [23], intertube or interlayer $s p^{3}$ bonding in single-walled CNT bundles is not observed up to a stress level of $20 \mathrm{GPa}$, and the pressure treatment of single-wall CNTs to $62 \mathrm{GPa}$ did not produce an after-pressure-release superhard carbon phase [22]. The Raman spectra of unloaded single-walled CNTs do not depend on pressure treatment up to 24-34 GPa, but nanoindentation of single-walled nanotubes (SWNTs) can produce forcedepth curves of that comparable to diamond in some experiments [24].

In this Letter, the transformation of the bonding structure in single-walled or multiwalled CNTs and graphite during nanoindentation is investigated from a mechanistic point of view using molecular dynamics simulation. A sharp rise in hardness occurs at $12 \mathrm{GPa}$ in graphite and at $16 \mathrm{GPa}$ in the CNTs, but the interlayer $s p^{3}$ bonds, which first form under a pressure of $36 \mathrm{GPa}$ in graphite and $46 \mathrm{GPa}$ in CNTs, are reversible until large-scale $s p^{3}$ hybridization occurs at a stress level of about $90 \mathrm{GPa}$. As the pressure increases during indentation, the $s p^{3}$ bonds at the center of the indentation zone are destroyed. The peak stress attained is about $120 \mathrm{GPa}$ in the CNTs, which is higher than the compressive strength of singlecrystal diamond that is lightly below $100 \mathrm{GPa}$ [25]. The compressive strength, i.e., the maximum load before failure, for graphite is $109 \mathrm{GPa}$.

In the present simulation, the second-generation reactive empirical bond order potential, developed for solid carbon on the basis of the Tersoff-Brenner expression [26], is used. The potential has been modified to specifically describe the interatomic interaction of carbon atoms in diamond and graphite lattices. In addition, non- 
local effects have also been incorporated via an analytic function. The capability of the potential to correctly describe the bond breaking and switching between multicarbon atoms [27-29] has been verified. A nonbonding interatomic interaction in the form of a Lennard-Jones 6-12 potential [30] has also been included. Simulations of the indentation of single-walled $(6,6)$, biwalled $(6,6) /(11,11)$, and triwalled $(6,6) /(11,11) /(16,16)$ CNTs with length of $98.38 \AA$ on substrates are performed. For comparison, a trilayered graphite sheet is simulated as well. The indenter used has a spherical apex of radius of $R=25 \AA$. Both the indenter and the substrate are simulated by virtual force via a repulsive potential [31] $V(r)=A \theta(R-r)(R-r)^{3}$ to avoid the interlinking with the carbon atoms, where $A$ is a force constant, $\theta(x)$ is the step function, $R$ is the indenter radius, and $r$ is the distance from the atom to the center of the indenter sphere. In this work, we take the force constant $A=$ $200 \mathrm{eV} / \AA$. In all simulations, the indenter is pressed at the center of the samples and penetrates with a speed of $5 \mathrm{~m} / \mathrm{s}$ at a constant temperature of $300 \mathrm{~K}$ controlled by the Berendsen scheme [32], and a time step of $1 \mathrm{fs}$ is used.

The nanohardness for graphite is presented in Fig. 1 as a function of the indentation depth. Here the nanohardness is defined as the indentation load divided by the contact area, which is determined from the number of atoms entering the repulsive virtual force field of the indenter. There are three distinctive regions in Fig. 1: from $a$ to $b$ (region I) is the soft linear region where the

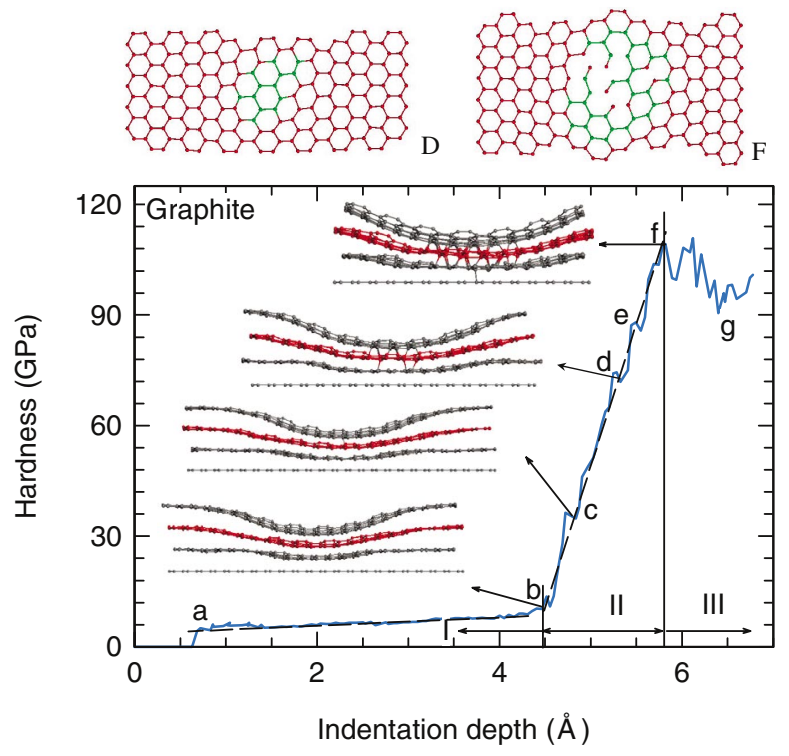

FIG. 1 (color). Hardness versus indentation depth curve of a graphite sample with three distinct regions: soft region I, hard region II, and unstable region III. The insets at points $b, c, d$, and $f$ on the curve show the geometry deformation and interlayer $s p^{3}$ bonding at the points. Insets $\mathrm{D}$ and $\mathrm{F}$ are the bonding conditions of atoms on the second layer of the sample where the red atoms are in a $s p^{2}$ state and the green ones are in a $s p^{3}$ state. hardness is low and increases slowly with the indentation depth with a modulus of about $10 \mathrm{GPa}$; the region from $b$ to $f$ (region II) shows the hard phase where the hardness increases sharply, but still linearly, with the indentation depth. The slope is 73 times that in region I, and represents a modulus of about $730 \mathrm{GPa}$, which is comparable to the corresponding value for diamond (700 to $1200 \mathrm{GPa}$ ) [33]. In the third region, from $f$ to $g$ (region III), the hardness becomes unstable, and the strength starts to drop. No interlayer bond forms, up to the transition point $b$, at which all the interlayer spaces of the graphite approach $2 \AA$ (see the inset at $b$ ). Beyond that, the interlayer interaction may be dominated by the Brenner potential and by the formation of the interlayer $s p^{3}$ bonds, particularly when the thermal motion of the atoms at $300 \mathrm{~K}$ is considered. The first interlayer bonding (bond length less than $1.7 \AA$ ) can be seen to occur at $c$, at a hardness of $36 \mathrm{GPa}$, following a small hardness drop (see inset). With increasing indentation depth, the hardness continues to increase to about $75 \mathrm{GPa}$, where another small drop in hardness is observed with the formation of more interlayer bonds (see inset at $d$ ) and a uniform $s p^{3}$ structure at the center of the second graphite layer (inset D). Beyond that, the hardness continues to increase to its peak value $109 \mathrm{GPa}$ at $f$, keeping the same overall steep slope, with some slight drops. At $f$, interlayer bonding occurs over a larger area, but at the center of the indented region, some $s p^{3}$ structures have been destroyed and an amorphous phase with a mixture of $s p^{2}$ and $s p^{3}$-bonded carbon atoms starts to appear (see inset $\mathrm{F}$ ). The area of amorphization increases with the indentation depth, until the whole structure finally becomes unstable. The uniform $s p^{3}$ phase formed at the pressure center of the graphite sample can bear ultrahigh compressive stress, up to $109 \mathrm{GPa}$ before destruction. Its strength is comparable to the compressive strength of diamond [25] and can explain why graphite under high-pressure can crack the diamond anvil [12].

However, if the pressure is removed before reaching a value of $74 \mathrm{GPa}$, all the $s p^{3}$ bonding structures return to the original $s p^{2}$ graphite layer structure and the sample resumes its soft phase. This result is consistent with experimental observations [10,11]. It should be noted that the $\sim 100 \mathrm{GPa}$ strength of the graphite and the diamond is obtained under a nonuniform compressive condition where shear stress may play important role. Under uniform compression, the pressure-induced $s p^{3}$ structure in a graphite sheet with periodic boundary condition is difficult to destructed even up to $\sim 1 \mathrm{TPa}$, but the softhard phase transition is nearly the same.

Nanoindentation of CNTs yields similar results. A typical hardness-indentation depth curve of a biwalled CNT is shown in Fig. 2. Two linear regions can be seen: the soft region $a-b$ and the hard region $c-d$. The small rise in the hardness in $b-c$ is due to the strength of the vertical $\mathrm{C}-\mathrm{C}$ bond of the inner tube, whose diameter is much smaller than the indenter tip radius. The slope is 


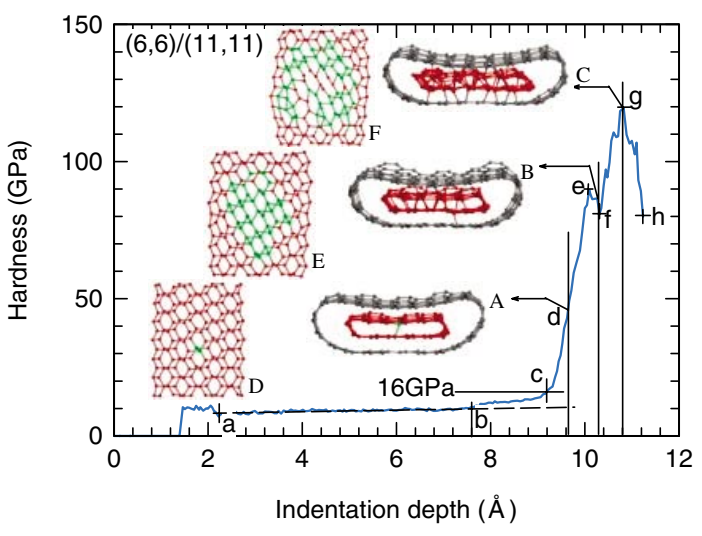

FIG. 2 (color). Hardness-depth curve of nanoindentation of the biwalled CNTs. The insets A, B, and $\mathrm{C}$ show the geometry and interlayer bonding at points $d, f$, and $g$ on the curve; the insets D, E, and F show the bonding conditions of atoms on the inner tube of the sample where the red atoms are in a $s p^{2}$ state and the green ones are in a $s p^{3}$ state.

$0.297 \mathrm{GPa} / \AA$ in section $a-b$ and $1.56 \mathrm{GPa} / \AA$ in section $b$-c. At point $c$, a sharp transition from the soft to the hard phase occurs, as all the interwall spaces approach $2 \AA$. The hardness at $c$ is about $16 \mathrm{GPa}$, higher than the critical value of $12 \mathrm{GPa}$ for graphite (point $b$ in Fig. 1). The slope of the hard phase is about 62 times of that in the soft section $b$-c. Interlayer bonding does not occur until the hardness reaches a value of $43 \mathrm{GPa}$ at $d$, where the first interlayer bonding is observed, as shown by insets A and $\mathrm{D}$ in Fig. 2. The $s p^{2}$ to $s p^{3}$ bonding transition is reversible when the unloading occurs before the applied pressure reaches a value of about $90 \mathrm{GPa}$. In this case, no permanent change occurs to the CNT after unloading. This is consistent with the experimental findings that show no change in electric property, hardness, or Raman spectra of CNTs after unloading from applied pressures of up to $65 \mathrm{GPa}$ [22-24]. Slightly beyond $90 \mathrm{GPa}$, large-scale interwall $s p^{3}$ bonds form and cause a drop in the hardness-depth curve from $e$ to $f$. Insets $\mathrm{B}$ and $\mathrm{E}$ show the interwall bonding and the uniform distribution of $s p^{3}$ atoms on the inner tube at point $f$. Further indentation beyond $f$ leads to a continuous increase in the hardness until the maximum at about $119 \mathrm{GPa}$ is reached at $g$. At the maximum pressure, the uniform $s p^{3}$ structure at the center of the indented region is destroyed and an amorphous $s p^{2}$ and $s p^{3}$ phase is obtained, as shown by Fig. 3 . Further indentation leads to the onset of instability, leading to the decrease of the hardness and, ultimately, to the complete destruction of the CNT.

Figure 4 shows the residual interwall bonding structure after unloading from point $f$ and point $h$ in Fig. 2. It is obvious that when the compressive stress is higher than $90 \mathrm{GPa}$, the interlayer bonding is irreversible. Comparing Figs. 4(a) and 4(b) with insets B and E of Fig. 2 indicates that all but a few of the interwall $s p^{3}$ bonds are broken during unloading. Well beyond the point of the maximum hardness on the indentation curve in Figs. 4(c) and 4(d),

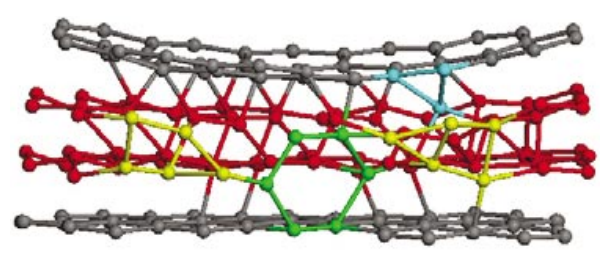

FIG. 3 (color). Snapshot of the middle part of the CNT from Fig. 2 at point $g$. The outer tube is in gray and the inner one is in red. Intermediate amorphous phase with a mixture of a hexagonal and a pyramidal structure is shown with interlayer hexagon in green, pentagon in yellow, and triangle in blue.

an amorphous phase with a structure formed from mixed $s p^{2}$ and $s p^{3}$ bonding is obtained after unloading (from point $h$, Fig. 2), indicating that the CNTs must be loaded well beyond $65 \mathrm{GPa}$ to produce a superhard amorphous phase of $s p^{2}$ and $s p^{3}$.

The indentation curves of single-walled and triwalled CNTs are compared with those of the biwalled CNT in Fig. 5. The maximum nanohardness of $121 \pm 2 \mathrm{GPa}$ remains almost the same in all three cases and is higher than that of the graphite sample (109 GPa) but comparable to the reported microhardness of single-crystal diamond, $\sim 100 \mathrm{GPa}$ [25], and 88 to $147 \mathrm{GPa}$ from the data base [33]. Similar to the biwalled tubes, no permanent change occurs to the triwalled CNTs after unloading from a compressive stress up to $93 \mathrm{GPa}$.

Detailed simulations show that when the force constant $A$ reduces to $20 \mathrm{eV} / \AA$, the hardness-depth curve for biwalled CNTs is nearly the same. When $A$ changes from 200 to $2 \mathrm{eV} / \AA$, the predicted strength has an increase less than $7 \%$. Increasing the indenter size from 25 to $50 \AA$ has a very weak influence on the hardness curve. The softhard phase transition is rather insensitive to the indenter size and hardness, and the boundary conditions and the
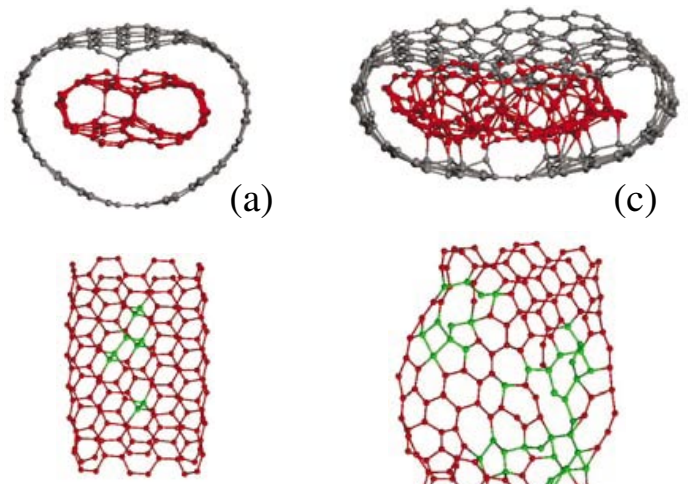

(b)

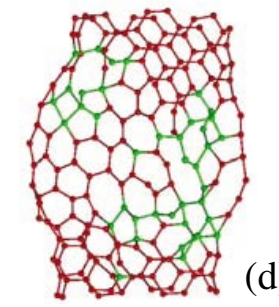

(d)

FIG. 4 (color). Geometry and permanent interlayer bonding structures of the biwalled CNT: (a),(b) after unloading from point $f$ in Fig. 2; (c),(d) after unloading from point $h$ in Fig. 2. (a) and (c) are the geometry of section in the middle part of the CNTs where the inner tube is in red and the outer one is in gray; (b) and (d) are the inner tube with $s p^{2}$ atoms in red and $s p^{3}$ atoms in green. 


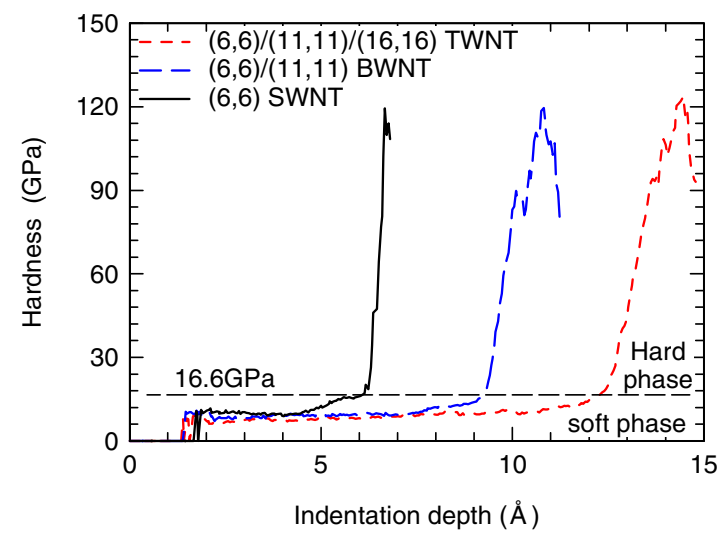

FIG. 5 (color online). Hardness versus indentation depth curves of single-walled CNT (SWNT), biwalled CNT (BWNT), and triwalled CNT (TWNT) with the inner tube of $(6,6)$, and all the tubes are armchair tubes.

number of layer and in-plane size of the sample graphite sheets.

In conclusion, both graphite and CNTs transform between soft and hard phases during indentation, with the transition compressive stress being about 12 and $16 \mathrm{GPa}$, respectively. In graphite, deeper penetration leads to the formation of interlayer $s p^{3}$ bonding, resulting in a nanohardness that increases linearly with the penetration depth with a modulus of about $730 \mathrm{GPa} / \mathrm{nm}$. The phase transition between the soft and hard phases is recoverable up to a compressive stress of $74 \mathrm{GPa}$, beyond which uniform $s p^{3}$ bonds form at the center of the indented region. At higher stresses some of the interlayer $s p^{3}$ become permanent and remain even after unloading. The nanohardness can reach $109 \mathrm{GPa}$ in the graphite sample, and about $120 \mathrm{GPa}$ in the CNTs, which is comparable to the reported microhardness of single-crystal diamond. These results may explain the seemingly paradoxical experimental observation that, while no increase in nanoindentation hardness can be detected in graphite and CNTs after-pressure treatment up to $65 \mathrm{GPa}$ $[10,11,22]$, graphite sheets can be hard enough to crack the superhard diamond anvil during a compressive test of over $23 \mathrm{GPa}$ [12], and CNTs may be as hard as a diamond during indentation [24].

The work is supported by the National Science Foundation of China, Croucher Foundation, and RGC (CA02/ 03.EG01) at the Hong Kong University of Science and Technology. It is also supported by a grant from the Hong Kong Research Grant Council (PolyU5312/03E).

*Corresponding author.

Electronic address: wlguo@nuaa.edu.cn

[1] R. F. Service, Science 281, 940 (1998).

[2] R. H. Baughman, A. A. Zakhidov, and W. A. de Heer, Science 297, 787 (2002).

[3] H. Hirai, K. Kondo, N. Yoshizawa, and M. Shiraishi, Appl. Phys. Lett. 64, 1797 (1994).
[4] M. E. Kozlov, M. Hirabayashi, K. Nozaki, M. Tokumoto, and H. Ihara, Appl. Phys. Lett. 66, 1199 (1995).

[5] H. Hirai, K. Kondo, M. Kim, H. Koinuma, K. Kurashima, and Y. Bando, Appl. Phys. Lett. 71, 3016 (1997).

[6] A. G. Lyapin, V.V. Brazhkin, E. L. Gromnitskaya, S.V. Popova, O.V. Stal'gorova, R. N. Voloshin, S. C. Bayliss, and A.V. Sapelkin, Appl. Phys. Lett. 76, 712 (2000).

[7] V.V. Brazhkin, A. G. Lyapin, S. V. Popova, Y. A. Klyuev, and A. M. Naletov, J. Appl. Phys. 84, 219 (1998).

[8] A. G. Lyapin, V.V. Mukhamadiarov, V.V. Brazhkin, S. V. Popova, M.V. Kondrin, R. A. Sadykov, E.V. Tat'yanin, S. C. Bayliss, and A.V. Sapelkin, Appl. Phys. Lett. 83, 3903 (2003).

[9] J. R. Patterson, S. A. Catledge, and Y. K. Vohra, Appl. Phys. Lett. 77, 851 (2000).

[10] J. R. Patterson, S. A. Catledge, Y. K. Vohra, J. Akella, and S. T. Weir, Phys. Rev. Lett. 85, 5364 (2000).

[11] J. R. Patterson, A. Kudryavtsev, and Y. K. Vohra, Appl. Phys. Lett. 81, 2073 (2002).

[12] W. L. Mao, H. Mao, P. J. Eng, T. P. Trainor, M. Newville, C. Kao, D. L. Heinz, J. Shu, Y. Meng, and R. J. Hemley, Science 302, 425 (2003).

[13] Y. Meng et al., Nat. Mater. 3, 111 (2004).

[14] T.W. Odom et al., Nature (London) 391, 62 (1998); J.W. G. Wildoer et al., Nature (London) 391, 59 (1998).

[15] T.W. Tombler et al., Nature (London) 405, 769 (2000); E. D. Minot et al., Phys. Rev. Lett. 90, 156401 (2003).

[16] H.W. Ch. Postma, T. Teepen, Z. Yao, M. Grifoni, and C. Dekker, Science 293, 76 (2001).

[17] A. Modi et al., Nature (London) 424, 171 (2003).

[18] A. Kis et al., Nat. Mater. 3, 153 (2004).

[19] J. Tang, L. Qin, T. Sasaki, M. Yudasaka, A. Matsushita, and S. Iijima, J. Phys. Condens. Matter 14, 10575 (2002).

[20] U. D. Venkateswaran, A. M. Rao, E. Richter, M. Menon, A. Rinzler, R. E. Smalley, and P. C. Eklund, Phys. Rev. B 59, 10928 (1999).

[21] J. Tang, L. Qin, T. Sasaki, M. Yudasaka, A. Matsushita, and S. Iijima, Phys. Rev. Lett. 85, 1887 (2000).

[22] J. R. Patterson, Y. K. Vohra, S.T. Weir, and J. Akella, J. Nanosci. Nanotech. 1, 143 (2001).

[23] S. Chan, W. Yim, X. Gong, and Z. Liu, Phys. Rev. B 68, 075404 (2003).

[24] M. Popov, M. Kyotani, and Y. Koga, Diam. Relat. Mater. 12, 833 (2003).

[25] Y. G. Gogotsi, A. Kailer, and K. G. Nickel, Nature (London) 401, 663 (1999).

[26] D.W. Brenner et al., J. Phys. Condens. Matter 14, 783 (2002).

[27] B. I. Yakobson, C. J. Brabec, and J. Bernholc, Phys. Rev. Lett. 76, 2511 (1996).

[28] J. A. Harrison, C.T. White, R. J. Colton, and D.W. Brenner, Surf. Sci. 271, 57 (1992).

[29] M. B. Nardelli, B. I. Yakobson, and J. Bernholc, Phys. Rev. B 57, R4277 (1998).

[30] Web site of Brenner's software, http://www.engr. ncsu.edu/mat/CompMatSci/projects.html.

[31] C. L. Kelchner, S. J. Plimpton, and J. C. Hamilton, Phys. Rev. B 58, 11085 (1998).

[32] H. J. C. Berendsen, J. P. M. Postma, W. F. van Gunsteren, A. DiNola, and J. R. Haak, J. Chem. Phys. 81, 3684 (1984).

[33] MatWeb, http://www.matweb.com/. 